\title{
5. DE LOS ESPAÑOLES Y EXTRANJEROS
}

(TíTULO I, CAP. I)

\author{
ELISA PÉREZ VERA \\ Catedrática de Derecho Internacional Privado \\ UNED
}





\title{
5. DE LOS ESPAÑOLES Y EXTRANJEROS
}

\author{
(TÍTULO I, CAP. I)
}

POR

ELISA PÉREZ VERA

Catedrática de Derecho Internacional Privado

UNED

\section{DE LOS ESPAÑOLES Y EXTRANJEROS \\ (Título I, Cap. I)}

Bajo este epígrafe genérico, la Constitución de 1978 contiene una serie de normas, en parte programáticas, sobre dos temas básicos en la construcción de todo Estado moderno. Se trata, de una parte, de la determinación de quiénes tienen la condición de nacionales (artículo 11) y, de otra, del trato que van a recibir aquellos que, por no ostentar tal condición. se consideran extranjeros (artículo 13).

En el segundo plano, el del trato a los extranjeros, el artículo 13, en sus apartados $3 .^{\circ}$ y $4 .^{\circ}$, incorpora además los principios rectores de dos instituciones, la extradición y el asilo, de naturaleza jurídica diferente, pero que hunden sus raices en un mismo presupuesto: la posibilidad de que la conducta humana sea enjuiciada de forma autónoma, y a veces encontrada, por cada uno de los Estados que integran la Comunidad internacional.

Así, mientras que la extradición, como expresión de la cooperación internacional en materia penal, se basa sobre una coincidente apreciación negativa de la conducta de la persona extraída, la figura del asilo hunde sus raíces en el deseo de proteger ciertos derechos fundamentales (básicamente, la vida y la libertad) de personas perseguidas en su país de origen, por motivos que el Estado que concede el asilo considera no sólo insufi- 
cientes, sino contrarios a los principios básicos en materia de protección de los derechos humanos.

I. Entre ambos artículos, el número 12 proclama que "Los españoles son mayores de edad a los dieciocho años». En comentarios, escritos al hilo de la adopción de nuestro texto constitucional, expresaba mis dudas sobre la ubicación sistemática de este precepto. En efecto, la mayoría de edad en nada afecta a la condición de español y, menos aún, a la de ningún extranjero, excluidos correctamente de su ámbito de aplicación por el propio tenor literal de la norma.

Ahora bien, y antes de ocuparnos de este aspecto "formal», conviene que nos pronunciemos sobre la necesidad y conveniencia de la norma misma. En este sentido, no cabe olvidar que, si bien la constitucionalización de la mayoría de edad es una novedad de la Constitución de 1978, no lo es el que la norma fundamental del Estado fije la edad a partir de la cual los nacionales pueden y deben participar en la vida política del país 1.

En tal contexto, y puesto que a lo largo del período constituyente se impuso la tesis favorable al reconocimiento de los derechos políticos a partir de los 18 años, de acuerdo con las tendencias imperantes en la órbita europeo-occidental, el texto terminó consagrando la mayoría a partir de tal edad. Solución correcta, ya que una postura intermedia que limitara el alcance de la norma constitucional a la edad habilitante para el ejercicio de los derechos políticos, podría traducirse en situaciones anómalas: por ejemplo, en que alguien con graves responsabilidades públicas pudiera, al mismo tiempo, encontrarse sujeto, en cuanto al ejercicio de sus derechos privados, a la patria potestad o a la tutela de un tercero ${ }^{2}$.

Dilucidado este extremo, la cierta falta de correspondencia entre el epígrafe que encabeza el capítulo I del Título I con el contenido del artículo 12, puede subsanarse fácilmente si se entiende que la norma que en él se contiene incorpora una precisión temporal sobre el momento en que la

1 Asi, p. ej., la Constitución española de 1931 fijaba en los artículos 36 y 53 , respectivamente, el derecho de sufragio activo y pasivo de los mayores de 23 años, mientras que el artículo 69 establecía que "Sólo serán elegibles para la Presidencia de la República los ciudadanos españoles mayores de cuarenta años..."

2 Observación válida, en general, aunque tal situación no se hubiera producido en el momento histórico que consideramos, ya que con anterioridad al referendum constitucional, el Real Decreto-ley de 16 de noviembre de 1978 (BOE del 17 de noviembre de 1978) modificó en igual sentido el Código Civil. Vid., también, Real Decreto-ley de 5 de diciembre de 1978 ( $B O E$ del 6 del mismo mes y año), por el que se modifica la Ley 50 de la Compilación navarra. 
condición de nacional alcanza su plena operatividad en relación con la vida política del país.

II. En relación con quiénes tienen la condición de nacionales, el artículo 11 incluye en su primer apartado una remisión a lo establecido por la ley, que será la que regule la adquisición, conservación y pérdida de la nacionalidad española (enumeración en la que ha de entenderse comprendida tambien la recuperación, que técnicamente puede conceptuarse como una readquisición de la nacionalidad).

En contraste con la situación relativa a la mayoría de edad, que por primera vez en nuestra historia se consagra en el texto constitucional, esta norma implica la parcial desconstitucionalización de la materia de nacionalidad. Una materia objeto de regulación constitucional en la tradición española. Ciertamente la aludida desconstitucionalización es sólo parcial en la medida en que, junto a la norma de remisión, el mismo artículo incorpora otras dos disposiciones sobre la nacionalidad que constituyen otros tantos mandatos al legislador en aspectos claves de su ordenación.

En cuanto al juicio que puede merecer dicha remisión, la doctrina ha sido unánime al aceptarla como la solución más adecuada, teniendo en cuenta que la complejidad del Derecho de la nacionalidad difícilmente puede abordarse en un texto constitucional. De hecho la normativa sobre la nacionalidad de las distintas Constituciones españolas coexistió pacificamente con su regulación en el Código civil, que incorporó sucesivamente los distintos criterios constitucionales.

Por lo demás, ha de admitirse que el desarrollo del Derecho de la nacionalidad, a partir de tales criterios, nọ tiene por qué ser necesariamente univoco. Situación que se acentúa hoy en España dado el limitado contenido normativo de los preceptos de la Constitución en la materia, referidos, de modo exclusivo, a la pérdida y a la doble nacionalidad. Que esto es así lo demuestra el que, con posterioridad a la Constitución de 1978, dos Leyes, de 1982 y 1990 respectivamente, han modificado los artículos pertinentes del Código civil, introduciendo dos reglamentaciones distintas de la nacionalidad, ambas respetuosas de las previsiones constitucionales.

Desde mi punto de vista, lo que en uno y otro caso es de lamentar es que no se decidiera elevar el rango de las leyes sobre nacionalidad a la categoría de Ley Orgánica, a partir de una interpretación literal del artículo 81 de la Constitución que no ha tenido en cuenta que el status de nacional es una situación jurídica fundamental, en cuanto presupuesto 
subjetivo para el ejercicio de los derechos fundamentales ${ }^{3}$. Bajo esta ópti$\mathrm{ca}$, hay que considerar, además, que si bien el Derecho de la nacionalidad no debe quedar fijado en una Constitución rígida, tampoco es deseable que quede sujeto a cambios demasiado frecuentes. Ahora bien, esta consideración crítica sobre el desarrollo legal de la Constitución, en nada afecta al propio contenido de nuestra norma fundamental.

Siguiendo el orden del artículo 11, su segundo apartado contiene una norma material del siguiente tenor: "Ningún español de origen podrá ser privado de su nacionalidad». Norma que se explica por sí misma, siempre que se tenga clara la distinción entre pérdida y privación de la nacionalidad. En efecto, si la primera se relaciona con el derecho a cambiar de nacionalidad, consagrado internacionalmente ${ }^{4}$, la segunda es consecuencia de un acto del Estado que se produce al margen o contra la voluntad del individuo.

La norma, que enlaza con la mejor tradición española, tal y como se recoge en nuestro Código Penal, y con la normativa internacional sobre los derechos humanos, merece un juicio positivo. En cuanto a su consagración solemne en la Constitución, tal vez haya que relacionarla, a contrario, con el hecho de que el artículo 20 del derogado Fuero de los Españoles previera la pérdida de la nacionalidad, por delito de traición, de cualquier español, con independencia de que lo fuera de modo originario o derivativo.

A su vez, el apartado tercero del artículo 11 contiene dos normas básicas en relación con el régimen de la doble nacionalidad. En efecto, mientras que el primer inciso plasma una norma-marco inspirada en la política convencional desarrollada con anterioridad, al amparo de lo dispuesto en el artículo 22 del Código civil (en la redacción que recibiera por Ley de 15 de julio de 1954), el segundo incorpora una norma directa que permite a los españoles naturalizarse en los países con los que España puede concertar tratados de doble nacionalidad (a los que se refiere la norma anterior), "sin perder su nacionalidad de origen».

Llegados a este punto, una primera constatación se impone: el ordenamiento jurídico español, al menos a partir de la Constitución de la Segunda República de 1931, considera a la doble nacionalidad como un instrumento válido para la aproximación entre los pueblos. Aceptado este hecho, que los debates en las Cortes Constituyentes ratifican, los proble-

3 Vid., en el mismo sentido, A. M. ${ }^{a}$ LóPEZ Y LÓPEZ, en Constitución española de 1978, t. II, dirigidos por Ó. Alzaga, Madrid, EDERSA.

4 Art. 15,2 de la Declaración Universal de Derechos Humanos de la ONU, de 10 de diciembre de 1948. 
mas que se podían plantear, en el marco del primer inciso, es decir, desde la perspectiva convencional, eran de una parte, cuál debería ser el alcance que los tratados internacionales atribuyeran a las dos nacionalidades; de otra, cuáles eran los países con los que «El Estado podrá concertar tratados de doble nacionalidad».

Sobre el primer punto nada nos dice el texto del artículo 11. Por lo demás, el tema tampoco aparece reflejado en los trabajos parlamentarios. En consecuencia, cabe suponer que en la negociación de estos convenios el Estado es libre, en principio, para acordar en cada momento los términos convencionales que más interesen a nuestra política exterior.

Ello implica, en mi opinión, que el Estado puede seguir el modelo a que responden los Convenios de doble nacionalidad concluidos con diferentes países latinoamericanos, que parten del supuesto de base de que «no hay ninguna objeción jurídica para que una persona pueda tener dos nacionalidades, a condición de que sólo una de ellas tenga plena eficacia» 5. De hecho, el Convenio hispano-colombiano de nacionalidad fue firmado y ratificado con posterioridad a la entrada en vigor de la Constitución de $1978^{6}$.

Pero supone tambien que el Estado puede concertar tratados que, al margen de que se denominen de doble nacionalidad (término que ya no recoge el Convenio hispano-colombiano que acabamos de citar), se traduzcan en la coexistencia de la nacionalidad española con otra u otras nacionalidades, siempre que pertenezcan a países de los contemplados en el ámbito de aplicación de la norma contenida en el inciso primero del artículo 11,3 . Coexistencia que, insisto, puede revestir la forma tradicional de los convenios con países latinoamericanos o cualquier otra que se estime más adecuada. Sobre este punto volveremos después.

En contraste con el silencio de la norma y de los trabajos parlamentarios sobre el contenido de los convenios de "doble nacionalidad", por utilizar la terminología acuñada, la determinación de los países con los que estos convenios pueden concluirse fue objeto de amplios debates, concretándose finalmente en ulos paises iberoamericanos $0 .$. aquellos que hayan tenido o tengan una particular vinculación con España». La apertura que esta formulación da al precepto se encuentra fuera de toda discusión; y es que, por esta vía cualquier evolución de nuestras relaciones interna-

5 Preámbulo del Convenio hispano-chileno de 24 de mayo de 1958 ( $B O E$ de 14 de noviembre de 1958). El subrayado es mío.

6 La firma tiene fecha de 27 de junio de 1979 y la ratificación es de 7 de mayo de 1980 (BOE de 28 y 29 de noviembre de 1980 ). 
cionales podrá ser tenida en cuenta, también desde la perspectiva de la doble nacionalidad.

El recurso a los trabajos parlamentarios muestra que la interpretación que proponemos no excede la intencionalidad de las Cortes Constituyentes. En efecto, la enmienda que condujo a la extensión del ámbito de aplicación de la norma a los países que tengan una particular vinculación con España, fue defendida alegando que "no debemos limitar el futuro, ya que es muy posible que si el movimiento de integración europeo continúa, y si esta Constitución dura mucho tiempo... creemos una situación en la que sea posible un estatuto de ciudadanía europeo y no tengamos que corregir la Constitución para dar este paso que está quizá en la historia y que sin duda está en el deseo de todos" ?

La aceleración del proceso de integración europea hace que hoy nos encontremos ante la hipótesis evocada en 1978. En efecto, el Tratado de Maastricht sobre la Unión Europea, firmado el 7 de febrero de 1992, consagra su segunda parte al establecimiento y regulación de la que denomina Ciudadanía de la Unión.

Ahora bien, todo estudio comparado entre el régimen de doble nacionalidad que se deriva de los Convenios concertados por España con paises latinoamericanos y la situación planteada por la coexistencia de la nacionalidad española con la futura ciudadanía europea, ha de partir de la diferencia fundamental que supone el que ésta se inserta en el contexto de un proceso de integración, frente al escaso significado jurídico de la Comunidad de países hispanoamericanos, sólidamente basada en lazos históricos y culturales, pero que no ha pasado hasta la fecha del plano de las declaraciones políticas.

En sintesis, los Tratados de doble nacionalidad concertados con países latinoamericanos establecen, todos ellos, un régimen que sólo funciona a instancia de un nacional de uno de los dos Estados partes (se trata de tratados bilaterales) que, previamente, para la adquisición de la nacionalidad del otro Estado parte ha de reunir los requisitos fijados por cada legislación interna para la adquisición de la nacionalidad (excepción hecha del sistema más favorable que establece el Convenio hispano-guatemalteco de 28 de julio de 1961).

En cuanto a sus consecuencias, los Convenios de doble nacionali-

7 Intervención del Sr. Morán López. Comisión de Constitución, en Constitución Española. Trabajos Parlamentarios (en adelante C.E.T.P.), Madrid, 1980, tomo III, pág. 3176. 
dad no prevén, en sentido estricto, una coexistencia de nacionalidades. $Y$ es que, en ellos se parte de la hipótesis, ya señalada, de que sólo una de las nacionalidades será efectiva, manteniéndose la otra "hibernada", es decir, carente de operatividad.

Por su parte, la ciudadanía europea se concibe como el nexo que une a la población de los Estados miembros con la Unión Europea, realidad nueva llamada a coexistir, sin desplazarla, con plural realidad estatal. No es, pues, casual que el Tratado de Maastricht sólo aborde el tema de la ciudadanía tras prever la creación de la Unión Europea que, precisa el Tratado, "tiene su fundamento en las Comunidades Europeas completadas con las políticas y formas de cooperación establecidas» que en él mismo se establecen.

Una primera consecuencia de esta construcción es que la ciudadanía de la Unión se extiende a utoda persona que ostente la nacionalidad de un Estado miembro", sin que se necesite para su obtención ninguna manifestación de voluntad del individuo concreto, ni se contemple la posibilidad de que el nacional de un Estado miembro se oponga a que le sea atribuida.

En cuanto a las relaciones de la ciudadanía con los vínculos nacionales preexistentes, del Tratado de Maastricht se deduce que los nacionales de los Estados miembros de la Unión Europea mantienen sin alteraciones su nacionalidad originaria, a la que se añadirá la condición de ciudadanos de la Unión que les hace titulares ude los derechos y sujetos de los deberes" previstos en el Tratado.

Derechos que se resumen en los siguientes términos: a) libertad de circulación y residencia en el territorio de los Estados miembros: $b$ ) derecho a ser elector y elegible en las elecciones municipales del Estado miembro en el que resida, a partir del 1 de enero de 1995; $c$ ) igual derecho en las elecciones al Parlamento Europeo, a partir del 1 de enero de 1994; d) derecho a acogerse a la protección de las autoridades diplomáticas y consulares de cualquier Estado miembro, en el territorio de un tercer país en el que no esté representado el Estado miembro del que sea nacional, a partir tambien del 1 de enero de 1994; e) derecho de petición ante el Parlamento Europeo, y $f$ ) derecho a dirigirse al Defensor del Pueblo, instituido en el mismo Tratado.

En suma, hay que reconocer que el sistema previsto en el Tratado de Maastricht presenta diferencias sustanciales respecto de los sistemas de doble nacionalidad convencional conocidos hasta ahora por el ordenamiento español. Ahora bien, estas diferencias no deben obstaculizar, en mi opinión, la calificación como convenio de doble nacionalidad del capí- 
tulo dedicado al tema en dicho Tratado. "Doble nacionalidad" que se traduce en la coexistencia de la nacionalidad española con la ciudadanía europea, dos nociones autónomas en que sólo la primera implica a la segunda. Es decir, los españoles, por serlo, seremos ciudadanos europeos, sin que ello signifique que todo ciudadano europeo sea español. $Y$ es que, la ciudadanía europea responde a una construcción política y jurídica autónoma que ha de entenderse en sus propios términos.

Un comentario mucho más breve requiere la norma incorporada en el segundo inciso del apartado tercero del artículo 11. Recordemos que en el mismo se consagra la posibilidad de que los españoles conserven su nacionalidad de origen, aunque se naturalicen en uno de los países con los que España puede concertar tratados de doble nacionalidad. Consagra así nuestro texto constitucional una excepción al principio que deduce, en determinadas condiciones, la pérdida de la nacionalidad española de quienes adquieren voluntariamente otra nacionalidad.

De este modo, desde la misma Constitución, se asume la existencia de dobles nacionales a los ojos del Derecho español que pueden no serlo para el otro país concernido (por ejemplo, si como nuestro propio Derecho, condiciona la adquisición de su nacionalidad a la renuncia a la anterior, sin excepciones). Ahora bien, hay que aceptar que tales situaciones son consecuencias normales de la existencia de un amplio margen de competencia discrecional de los Estados en materia de nacionalidad.

El carácter abierto de la fórmula utilizada por la Constitución para designar los países con los que España puede concertar tratados de doble nacionalidad suscitó el problema de saber en qué países podían naturalizarse los españoles sin perder su nacionalidad originaria. El tema se resolvió por la Ley 51/1982, de 13 de julio, de modificación de los artículos 17 al 26 del Código civil, que los concretó en los países iberoamericanos, Andorra, Filipinas, Guinea Ecuatorial y Portugal; enumeración que no ha sido modificada por la Ley $18 / 1990$, de 17 de diciembre.

En este contexto, cabe preguntarse si la ratificación del Tratado de Maastricht no debería ir acompañada de una nueva redacción del Código civil que extendiera tal posibilidad a la adquisición de la nacionalidad de los restantes Estados de la Unión Europea, además de a Portugal. Personalmente, estimo que sí, aunque pueda dudarse de la utilidad práctica de tal medida, si consideramos los derechos de todo tipo que comporta la ciudadanía europea.

III. Las normas básicas que han de presidir la ordenación del régimen jurídico aplicable a los extranjeros se recogen, como ya se ha indica- 
do, en el artículo 13. En efecto, dado que ningún Estado ha tenido un ámbito universal, la existencia de personas ajenas al mismo ha planteado, como constante histórica, la necesidad de determinar cuál será su actitud frente a quienes no gozan del status de nacional, es decir, frente a los extranjeros.

El apartado primero del artículo citado enuncia la norma de base sobre la que se construye el sistema, al disponer que uLos extranjeros gozarán en España de las libertades públicas que garantiza el presente Título en los términos que establezcan los tratados y la ley". Asi pues, el punto de partida en el tratamiento de la extranjería - no habiéndose planteado en ningún momento la exclusión de derechos de los extranjeros y descartado el principio de reciprocidad como eje de su reconocimiento-, es el de la asimilación a los nacionales, matizada por lo que dispongan los tratados y la ley.

En este punto, he de reconocer que los temores que compartí con parte de la doctrina de que el desarrollo de la norma constitucional plasmara en un régimen abiertamente discriminador frente a los extranjeros, no se han visto confirmados en la práctica. Una práctica en la que, junto a la denominada Ley Orgánica de extranjería de 1985, tienen especial relevancia las decisiones del Tribunal Constitucional ${ }^{8}$ y la actuación del Defensor del Pueblo.

El segundo apartado del artículo 13 trata de los derechos políticos y del acceso a las funciones y cargos públicos, es decir, de un campo abonado para las mayores discriminaciones en el seno de la organización estatal. De ahí que la norma se formule como una excepción al principio de base. En efecto, el párrafo se inicia con la siguiente frase: "Solamente los españoles serán titulares de los derechos reconocidos en el artículo 23...".

Respecto de los derechos políticos en sentido estricto, las razones para limitar su goce a los nacionales hunde sus raíces en el concepto mismo del Estado como organización, sobre base territorial de la población que lo integra. En cuanto a la exclusión de los extranjeros del acceso a las funciones y cargos públicos, es una constante de nuestro Derecho. Ahora bien, tal exclusión no puede equivaler a que se prohiba a los extranjeros el ejercicio de determinadas actividades realizadas a veces, e incluso normal-

8 Vid., especialmente, las sentencias 99/1985, sobre libertades de los extranjeros en España, y 115/1987, recaída en el recurso de inconstitucionalidad promovido por el Defensor del Pueblo contra la Ley Orgánica 7/1985, de 1 de julio, sobre derechos y libertades de los extranjeros en España. 
mente, por funcionarios. En efecto, en este ámbito, creo que la Constitución lo que reserva a los españoles no es el desarrollo de actividades de determinadas, sino el "título" con el que se ejercen.

La norma que comentamos se completa, además, con lo podríamos calificar de "excepción a la excepción". Y es que el propio precepto contempla la posibilidad de que se reconozca a los extranjeros el derecho de sufragio activo en las elecciones municipales, en virtud de lo que, atendiendo a criterios de reciprocidad, pueda establecerse por tratado o ley. En mi opinión, estamos ante uno de los rasgos más internacionalistas de la Constitución de 1978. Aún más, creo que en este contexto resulta positivo el recurso a la noción de reciprocidad que puede contribuir, a medio y largo plazo, a mejorar la vida diaria de los emigrantes españoles; $y$ es que, por tal vía, parece más fácil que nuestros emigrantes lleguen a convertirse en sujetos políticamente activos de la comunidad en que se encuentran insertos, logrando así que sus intereses sean mejor atendidos.

Hasta aquí la explicación satisfactoria del funcionamiento del párrafo segundo del artículo 13 en una sociedad internacional construida sobre la idea de cooperación. Veamos ahora hasta qué punto puede considerarse compatible el enunciado constitucional con la noción de ciudadanía europea $y$, en concreto, con el derecho de los ciudadanos europeos, que no tengan la nacionalidad española, a ser elegidos en las elecciones municipales.

La respuesta no ha de buscarse sólo, ni siquiera básicamente, en este Título Primero, Capítulo Primero, de la Constitución, sino que hay que considerar la regulación de los Tratados Internacionales contenida en los artículos 93 a 96 del mismo texto constitucional. El comentario de estos artículos corresponde a otra ponente; no obstante, sí que hay que recordar aqui que el artículo 93 recoge, como no podía ser menos dado el momento de su redacción, la posibilidad de celebrar, con garantías parlamentarias reforzadas, Tratados "por los que se atribuya a una organización o institución internacional el ejercicio de competencias derivadas de la Constitución".

Esta disposición, destinada básicamente a facilitar la adhesión española al proceso de integración europeo, ha de interpretarse de forma dinámica, es decir, extendiendo su aplicación a todos los supuestos en que se produzca la situación descrita. Ahora bien, ¿el reconocimiento del derecho de sufragio pasivo a los ciudadanos europeos, que no sean españoles, se incluye en esta categoría? En mi opinión, sí.

Como ya he indicado, la configuración de la ciudadanía de la Unión corresponde a la dinámica propia del proceso de integración comunitaria, 
en el que España participa activamente. $Y$ es en este contexto en el que ha surgido una nueva noción, la ciudadanía de la Unión, que rompe el esquema binario (nacionales-extranjeros) sobre el que se construyó nuestra Constitución. El que estos ciudadanos europeos gocen, en vitud en una norma convencional ratificada por el procedimiento reforzado del artículo 93, de algunos de los derechos que la Constitución reservó a los españoles, en contraposición de los extranjeros, no me parece que exija la reforma de una norma que en su conjunto sigue siendo válida. Tanto más si consideramos que la definición de quiénes son españoles es una competencia que corresponde establecer a la Ley (ley ordinaria, como ya ha quedado señalado), de acuerdo con las directrices constitucionales ya examinadas.

En distinto plano, la exigencia del Tribunal de Justicia de las Comunidades Europeas de que los Estados deben eliminar situaciones de incertidumbre derivadas de la incompatibilidad, aún formal, entre los derechos internos $y$ el comunitario, se cumpliría modificando simplemente la normativa electoral. No obstante, he de reconocer que desde esta perspectiva puedo entender a quienes propugnan una reforma constitucional que, si se aborda, debería referirse al primer inciso de esta norma, es decir, a la reserva solamente a los españoles de los derechos reconocidos en el artículo 23. En tal hipótesis una posible redacción alternativa sería la siguiente: «Solamente los españoles y los ciudadanos de la Unión Europea, en los términos que fije el Derecho comunitario, serán titulares de los derechos..." (el resto, igual).

Por el contrario, una reforma que se circunscribiera a la excepción aplicable por hipótesis a todos los extranjeros, para incluir el «derecho de sufragio activo y pasivo en las elecciones municipales", no resolvería el tema de la participación de los ciudadanos de la Unión en las elecciones al Parlamento Europeo y resultaría previsiblemente insuficiente a corto o medio plazo, abriendo sin embargo la vía de unas reformas constitucionales que, en mi opinión, deberían evitarse siempre que fuera posible.

En efecto, considero que la mayor virtud de cualquier Constitución es su misma existencia. Aún más cuando, como en el caso de la Constitución Española de 1978, se redactó con un espíritu de consenso difícilmente reproducible. En tal sentido, no puedo apoyar la idea de una reforma que me parece técnicamente innecesaria.

Pocos comentarios voy a hacer a la norma contenida en el apartado tercero del artículo 13, referida a la extradición. En la misma destaca, en primer lugar, la consagración del principo de legalidad en cuanto a las fuentes de la extradición, ya que usólo se concederá en cumplimiento de un tratado o de la ley, atendiendo al principio de reciprocidad". Recorde- 
mos, en este punto, que la regulación interna española se recoge en la Ley de Enjuiciamiento Criminal y en la Ley 4/1936, de 21 de marzo, de extradición pasiva. Por otra parte, España ha ratificado, en 1982, el Convenio Europeo de Extradición de 13 de diciembre de 1957 y, en 1985, sus dos Protocolos adicionales de 15 de octubre de 1975 y 17 de marzo de 1978, respectivamente.

En segundo lugar, la norma que consideramos delimita su ámbito de aplicación, al señalar que "Quedan excluidos de la extradición los delitos políticos, no considerándose como tales los actos de terrorismon. Teniendo en cuenta lo difícil que puede resultar la precisión del concepto de delito político, un amplio sector doctrinal criticó que la Constitución incluyera el inciso que precisa que no se consideran como tales los actos de terrorismo. Ahora bien, al margen las dificultades concretas de calificación, la disposición me parece adecuada. Por una parte, las dificultades aludidas deben verse aliviadas por la ratificación española, en 1980, del Convenio Europeo para la represión del terrorismo de 27 de enero de 1977.

Un último punto a considerar es el silencio del texto constitucional sobre la exclusión de la extradición cuando el sujeto requerido sea nacional español. Tal exclusión estaba recogida en la Ley de extradición de 1958 , como lo está en la actualmente vigente de 1985. Lo que vendría a abonar la opinión de los profesores COBO DEL ROSAL y BOIX REIG, en el sentido de que pudiera llegarse a un reconocimiento constitucional de dicho principio, "en base a una interpretación sistemática del artículo 13", ya que "es sostenible que el indicado artículo estuviese pensado fundamentalmente para concretar determinados derechos de los extranjeros, por lo que, en consecuencia, la regulación de la extradición pasiva no comprendería al nacional»" .

Finalmente, el apartado cuarto del artículo 13 se ocupa del derecho de asilo. Se trata, en mi opinión, de una de las normas de nuestra Constitución con un proceso de elaboración más irregular. En efecto, de los trabajos parlamentarios resulta que el texto aprobado en el Pleno del Congreso el 6 de julio de 1978 (del siguiente tenor, "Se garantiza el derecho de asilo en los términos que la ley establezcan), no es el que se recoge en el Proyecto de Constitución aprobado por la misma Cámara el 21 de julio, en el que, por el contrario, figura la versión que la norma había recibido en la Comisión de Asuntos Constitucionales. Versión contra la que se habían manifestado los representantes de todos los Grupos Parlamentarios, a excepción de UCD y AP, por estimar que, con la

9 En Constitución Española de 1978, op. cit., pág. 252. 
fórmula utilizada, "el derecho de asilo ya no existe, constitucionalizado como tal» ${ }^{10}$.

No obstante, ese fue el texto que debatirá el Senado y el que, extendido a los apátridas, pasará a la Constitución que, en este punto dispone que "La ley establecerá los términos en que los ciudadanos de otros países y los apátridas podrán gozar del derecho de asilo en España». Dado el tenor literal de la norma, se ha podido escribir, no sin razón, que «el derecho de asilo ha quedado, pues, virtualmente vaciado de contenido, ya que su amplitud y su propia existencia dependerá por completo de la ley que lo regule»" ${ }^{11}$.

Con posterioridad, la Ley 5/1984, de 25 de marzo, reguladora del derecho de asilo y de la condición de refugiado y su Reglamento de aplicación (R.D. 511/1985, de 20 de febrero), aportan el adecuado desarrollo legal al apartado 4 del artículo 13 de la Constitución. Además, hay que tener en cuenta que España es parte del Convenio sobre el estatuto de los refugiados, de 28 de julio de 1951 y de su Protocolo de 31 de enero de 1967 (BOE de 21 de octubre de 1978). Textos todos ellos que demuestran que la España democrática ha asumido, como no podía ser menos, la cuota de solidaridad internacional que exige la situación de asilados y refugiados. Pese a todo, sigo considerando lamentable que la remisión global a la ley que establece nuestra vigente Constitución someta la suerte de un instrumento humanitario básico a los imprevisibles vaivenes de la vida política española.

IV. A modo de conclusión. Dado que el objeto de este Congreso es la valoración de la funcionalidad de nuestro texto constitucional, de las consideraciones que proceden puede deducirse que, en mi opinión, la Constitución de 1978, en el ámbito de materias que me ha correspondido comentar, ha funcionado globalmente bien, incluso mejor de lo que en el momento de su adopción algunos esperábamos.

Ello significa, evidentemente, que los artículos 11,12 y 13 de nuestra norma fundamental no sean susceptibles de críticas. Algunas se avanzan en esta ponencia $y$, desde otras perspectivas, cabrian sin duda otras más.

Ahora bien, creo que sería un error confundir un texto legal con un

10 Intervención del Sr. Roca Junyent, en C.E.T.P., op. cit., pág. 983.

11 P. AbARCA JunCo: "Nacionalidad y extranjería en la nueva Constitución", en Lecturas sobre la Constitución Española, Madrid, UNED, 1978, págs. 388-389. 
manual de Derecho; por ello considero que las críticas que pueden hacerse en base teórica no tienen por qué traducirse en propuestas de cambios. Por otra parte, por las razones expuestas y por otras que podrían extraerse del Título III del Capítulo III de la misma Constitución, tampoco creo que la ratificación del Tratado de Maastricht requiera una reforma del artículo 13.2 que reserva a los españoles determinados derechos, y que considero compatible con la atribución de fuente comunitaria de algunos de estos derechos a quienes gocen de la ciudadania europeo.

En consecuencia, no seré yo quien sugiera alteraciones en un conjunto normativo que ha probado su operatividad, y al que deseo, para concluir, una larga y feliz vida, que facilite la conviencia "de los españoles y los extranjeros".

Madrid, 31 de marzo de 1992 